\title{
An Ehanced Digical Character Recogniton using Art Network Classifier
}

\author{
Kapil Juneja \\ Department of Computer Science \& Applications \\ Maharshi Dayanand University \\ Rohtak 124001, Haryana, Rohtak
}

\author{
Nasib Singh Gill \\ Department of Computer Science \& Applications \\ Maharshi Dayanand University, \\ Rohtak 124001, Haryana, India
}

\begin{abstract}
Image Classification is one of the major applications of Image processing to place all the related images in a separate group. In some Recognition and Identification applications, Image classification is used to reduce the size of processing dataset. In this present work, the image classification is used to perform the digital character recognition. In this work, A Soft computing technique called Art Network is been implemented through the classification process. The work is divided into two main stages named Training and Recognition process. During the Training Phase, the input imageset is processed and divided in $\mathrm{N}$ Classes based upon the tolerance ratio. The Eligibility criterion to specific class is decided by Feature analysis over the image. In second stage, the feature extraction over the input image is performed and based on featured value; the related class is been identified. Now one-to-one comparison is performed on that class to identify the class on which comparison is performed. The effectiveness to the work is estimated based on matching ratio.
\end{abstract}

\section{Keywords}

Art Network; Classification; Noisy; Recognition; Featured

\section{INTRODUCTION}

The Digital character identification[1] is the major research area used to identify or recognize the character images. These images can be in the form of hand written scanned text or the font based digital text. The applications come with the character recognition includes the number plate recognition[2], image to text conversion[3], capcha reader[4], etc. There are numbered of complexities associated with both kinds of a character recognition system. The handwritten character identification [5] having the problem such as distance between the characters, overlapping of text, depth of ink stroke, text alignment, etc. These kinds of systems having the main problem to perform the character separation for the documents or the text. The major applications of these kinds of identification system include signature verification[6], image to text conversion, etc.

The digital text having the problem respective of the font specification. The simpler the font type, more accurate the recognition process will be. The variation in the digital text will be in terms of font type, size, font style, etc. Another specification in both types of character recognition includes the language specification[7]. The recognition text can be either language specific or language independent. If the recognition process is language specific, it is defined under the natural language processing, and the intelligent system can be defined under the language specification. If the system is language independent, it is completely based on image pixels.
In this present work, the character recognition is demonstrated for the digital text to identify the digital alphabets. The work is based on the feature based analysis. The recognition systems itself have a number of properties along with standard architecture. The architecture includes the basic three components called Input Image, Image Dataset and Recognition process. The Input Image and the Image Dataset should have similarities in different ways shown in table 1 . The accuracy of the recognition system will depend on the number of matching properties for input image and image dataset. i.e.

\section{Recognition Ratio $\boldsymbol{\alpha}$ Number of Properties Matched}

Table 1: Required Similarity in Input and Dataset Image

\begin{tabular}{|l|l|}
\hline \multicolumn{1}{|c|}{ Property } & \multicolumn{1}{c|}{ Description } \\
\hline File Format & $\begin{array}{l}\text { Both images should be of same file format } \\
\text { such as jpg, bmp, png etc }\end{array}$ \\
\hline Size & Is the physical size of image such as $2 \times 2 \mathrm{~cm}$ \\
\hline Resolution & Resolution is in dpi given as 400x400 \\
\hline $\begin{array}{l}\text { Brightness and } \\
\text { Contrast }\end{array}$ & should be similar. \\
\hline Font & Include font name, style, text etc. \\
\hline Image Type & $\begin{array}{l}\text { Complete Image, Image part or the featured } \\
\text { image }\end{array}$ \\
\hline
\end{tabular}

When the input image or the dataset images are collected or extracted they can be slightly different respective to defined properties in table 1 . In such case before implementing the recognition process some preprocessing operations[8] are performed to improve these Property based similarity ratio. The Accuracy of the system is based on this preprocessing stage. During the preprocessing phase, the selection about the image will be drawn on which comparison will be performed i.e. complete image, partial image or the featured image. If the image type is partial image the image segmentation or the image part extraction algorithm will be implemented during the recognition process. In case of featured image, the algorithmic feature extraction approach is implemented. These kind of extract improve the efficiency of the system and also increase the fault tolerance for the system. The components of the preprocessing stage are shown in Fig. 1. 


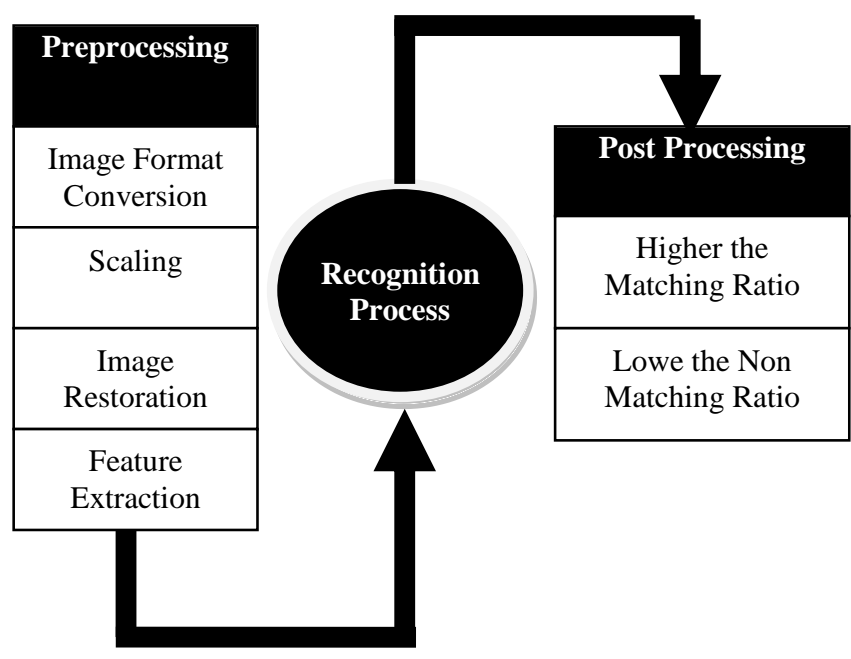

Fig. 1 : Components of Preprocessing

Where the preprocessing stage defines used to improve the effectiveness of the system in terms of efficiency and the accuracy. The post processing is used to estimate the reliability to the system. As discussed earlier, the reliability depends on two major factors. First, the matching ratio for correct character identification. Higher the ratio represents the perfectness of the system. The matching ratio is also tested under the noisy or distorted images. For a good identification system, the matching ratio should be more than $75 \%$ in case of noisy or noiseless images. The another post analysis factor is the ratio of non matching on invalid image. More the difference between matching and non-matching ratio, more effective the system will be. The components of the post processing are shown in Fig. 1. satisfactory even for a valid image, it means more work is required on the preprocessing phase.

In this presented system, the implementation is performed of same recognition architecture at the initial stage. However, the problem of this architecture is to perform the one-to-one match on each image from the image set. In this present work, the improvement on the efficiency is been suggested during the recognition phase by the inclusion of a classier on featured dataset. The detail of the presented architecture is given in section 3. To perform the classification on image set, the art network is been implemented in this paper. The description of the art network and the classification process is defined in section 2 . The Result analysis based on this proposed work is given in section 4 .

\section{ART BASED CLASSIFIER}

The classification is the one of major image processing application used to divide the available image set in separate groups called classes. To perform the classification process, at first need to define the number of classes and the eligibility criteria for each class. In this present work, a classifier is implemented on featured image. As the initial stage, the number of classes are defined and then some equational or mathematical Model to decide the eligibility criteria for the classification process. There are number of classes approaches suggested by different researchers such as neural network, fuzzy logic, regression analysis etc. In this present work, a specialized neural classifier called ART (Adaptive Resonance Theory) Network is defined.

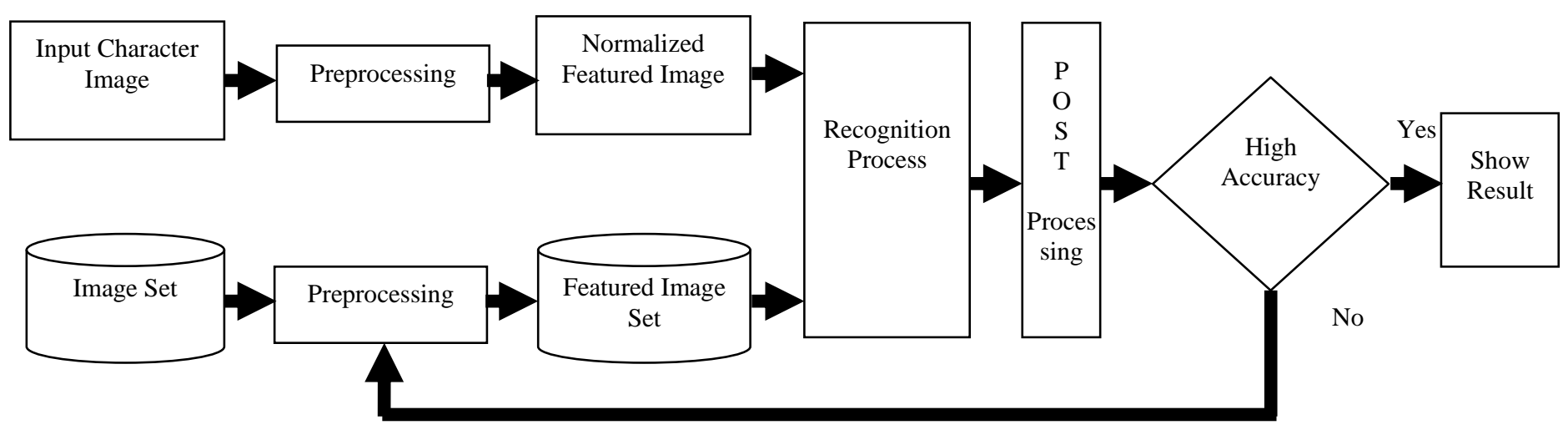

\section{Fig. 2 : Architecture of Digital Character Recognition}

The recognition process is the actual process to identify the matching ratio with all images over the dataset. The image that will give the highest matching ratio will be presented as the identified image. The basic architecture of the complete recognition process is shown in Fig. 2. The figure shows that the basic processing is performed on both the input image, and the data set separately but under same constraints defined in Fig.1. Once the preprocessing phase is done, and get the featured image set as well as feature input image with maximum similarity attributes. The recognition process will be performed on these featured images. The feature based one to one match of input featured image will be performed on each image of dataset. Based on the recognition process, most matched image will be identified. Now the matching ratio will be checked during the post processing phase to verify the accuracy level. If the accuracy is high and conclude the perfect match or mismatch, the result will be presented. However, if the matching ratio is not
ART network[9] is type of Artificial Neural Network used to perform categorization based on Pattern Analysis. The basic architecture of ART network is a layered architecture defined under linear units that represents the prototype vectors. Alike the other neural networks, the ART network performed the feature or pattern analysis during the training process. According to the basic training process of ART network, some weighted vectors are defined based on the similarity specification ratio. The input set in the ART network is defined in the form binary values or size n. Now based on the defined criteria the clustering process will be performed. Each learned cluster is defined by weighted vector $\mathrm{w}$. Based on these two parameters the normalized output is computed from the system. Let $\mathrm{x}$ is the input vector, $\mathrm{w}_{\mathrm{j}}$ is the weighted vector for cluster $\mathrm{j}$ then the normalized output vector for cluster $\mathrm{j}$ is given by $\mathrm{y}_{\mathrm{j}}$ given in equation (1). 


$$
y j=\frac{w_{j}^{T} x}{\left|w_{j}\right|^{2}}
$$

The training set is performed on the image set to carry out the featured classification. Once the training is performed, the second stage is to perform the verification process during the matching stage. The verification test is passed if it satisfies the equation (2).

$$
\frac{w i^{\mathrm{T}} \mathrm{x}}{|\mathrm{x}|^{2}}>\rho
$$

Here $\rho$ is defined as vigilance parameter. The output vector wi is called match with input vector $\mathrm{x}$, if a significant fraction of the 1 's in $\mathrm{x}$ identified in wi. The input value that satisfies the vigilance parameter of a specific cluster $\mathrm{j}$ is placed in that particular cluster or class.

\section{PROPOSED ARCHITECTURE}

The presented work is the improvement over the standard architecture of digital character identification by the involvement of a classifier. To perform the classification the ART network is been implemented on the featured image set just before the recognition process. The ART-CLASSIFIER- RECOGNIZER is shown in Fig. 5. The work of the classification process is about to reduce the dataset on which actual recognition process will be performed. The presented work is about to analyze the effect of classifier network in case of optical character recognition. The work is divided into two stages; the first work is to train the image using Art network and classify them according to image features. Here a good accuracy level is achieved while performing the classification. Once the images are trained the next work perform the recognition process. The analysis on faulty images is performed to check the accuracy level. The degree of acceptance of the input character for the database is identified here. The major algorithmic approaches used in this presented work are given in Fig. 3.
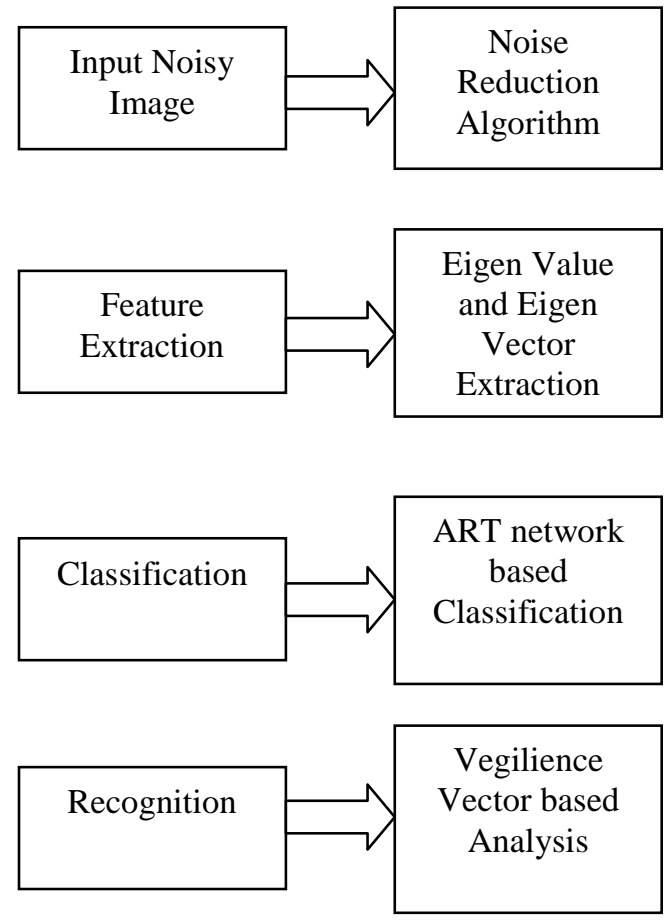

Fig. 3 : Algorithmic Processes
The presented work is been implemented under the extended architecture shown in Fig. 5, and the descriptive analysis on each stage is been defined here under.

\subsection{Input Image and ImageSet}

The presented work begins with specification of input image and the construction of image set. To perform the analysis; An image set of 26 alphabetic characters is taken. The basic parameters of this image set is shown in table 2 .

Table 2 : Image set Properties.

\begin{tabular}{|l|l|}
\hline Property & Value \\
\hline Number of Images & 26 \\
\hline Color & No \\
\hline Resolution & $20 \times 40$ pixels \\
\hline Image Format & Bmp \\
\hline Number of Instances & One Instance for One Font \\
\hline
\end{tabular}

The input image is also taken under same property values. However, the input image is tested under three criteria for getting the actual significance to the work. These parameters include the normal image, noisy image and noise rectified image. These parameters are shown in Fig. 4.

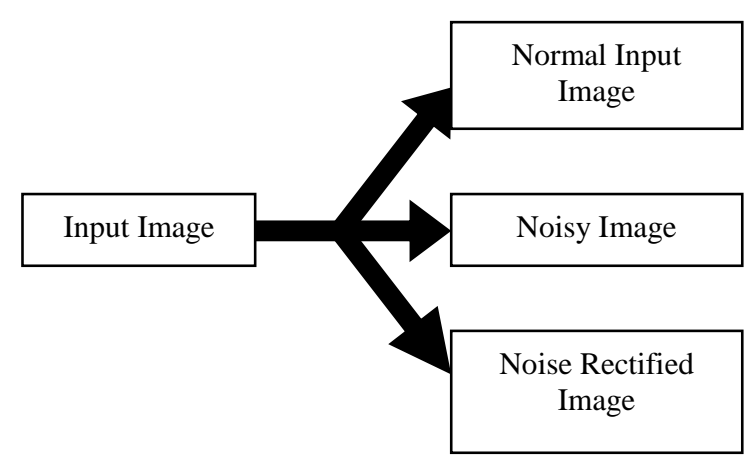

Fig. 4 : Different Forms of input Image

To make a noisy image, a randomly distributed noise is used over the image called Salt and Pepper Noise. The significance of work is checked by inclusion of different levels of the noise. The work is also been tested on the rectified image. The obtained accuracy in all three approaches is discussed in result section.

\subsection{Preprocessing}

As defined in section 1, the preprocessing stage itself is defined in the form of few sequential steps. The dataset and input image is identical in terms of physical size, resolution, color, contrast, etc. The image set is the identical dataset without any impurity in the image. However, the input image is either a normal character image or the noisy image. In case of noisy input image, Median filter is implemented to remove the noise from the image. Median filtering is done by, first sorting all the pixel values from the surrounding neighborhood into numerical order and then replacing the pixel being considered with the middle pixel value. Note that the median value must be written to a separate array or buffer so that the results are not. 


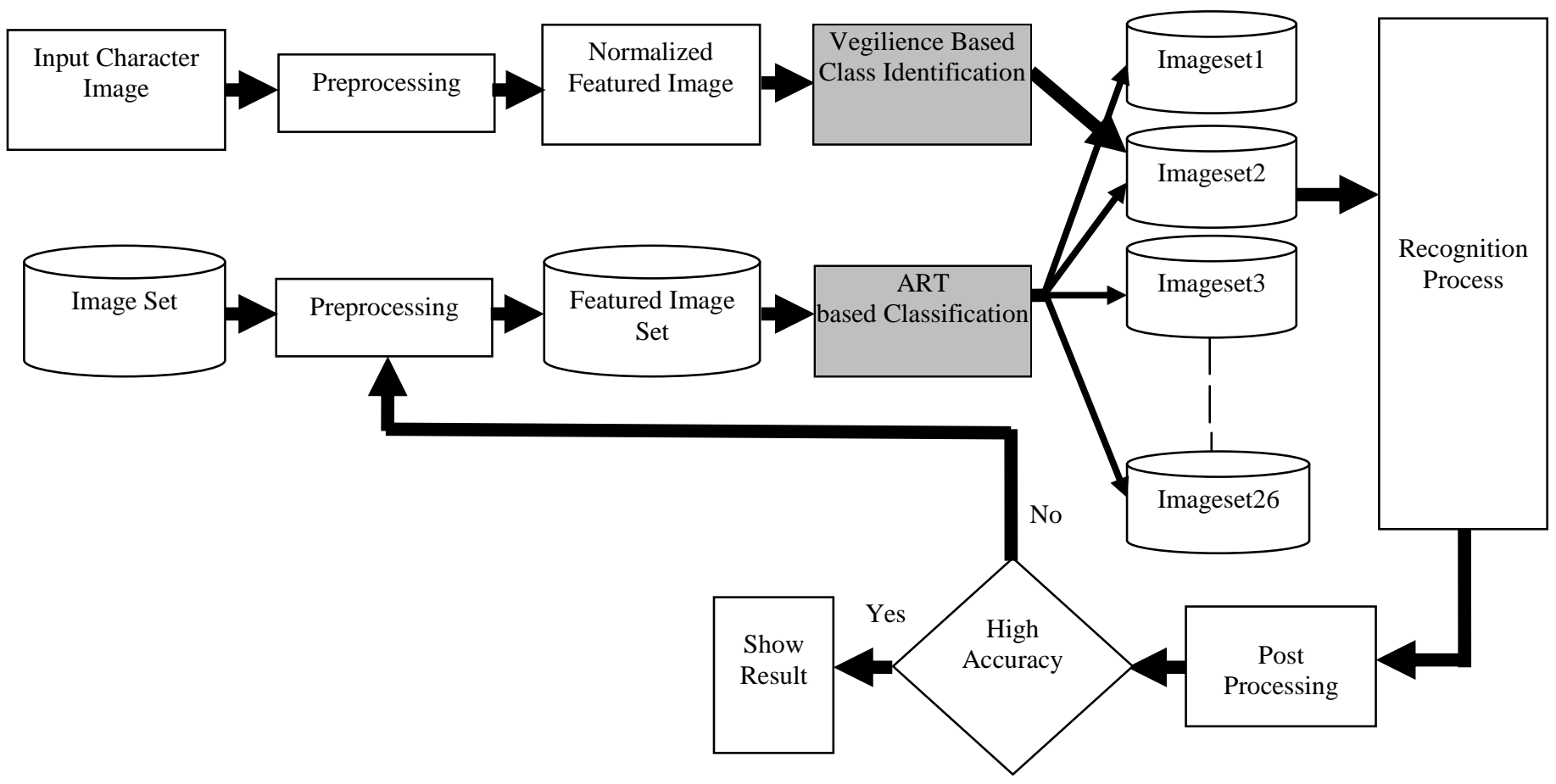

Fig. 5 : Proposed ART-CASSIFIER-RECOGNIZER

corrupted as the process is performed. The basic algorithm of Median Filter is shown as under MedianFilter(Img)

/*Img is the source input image infected from Salt \& Pepper Noise*/

\{

1. Define the Fileration window called nxn

2. Slide the Window over the and find median value on each window

3. Replace the window pixel by this median value.

4. Present the Result Image

\}

As the processing will be done, the normalized image will be obtained on the next work is about extract the feature from the image. In this work, the feature extraction is been performed based on eigen value and eigen vector based analysis. Once the eigen vector will be extracted from the image. It will construct a featured image set. Same operation of feature extraction will be performed on input image.

\subsection{Art Based Classificaton}

In this presented model, the main stage is the involvement of the ART based classifier to reduce the size of processed dataset during the recognition process. The basic process of ART is defined in section 2 . The algorithmic process followed is given as under.

\section{Traing(Imgs)}

/* Imgs is the featured image Set on which the raining process is performed $* /$

\footnotetext{
\{

1. Read Each Image from database Imgs one by one called Image I

2. Implement the Art Network for Image I

3. Define Vigilence Parameter V
}

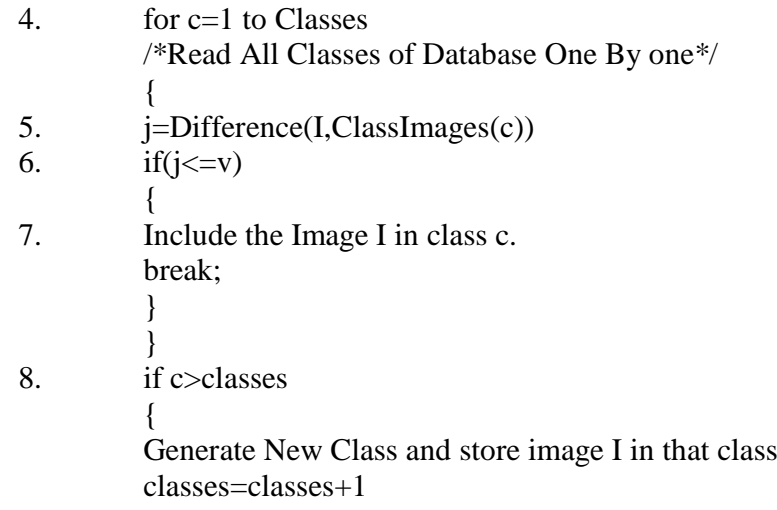

\subsection{Recognition}

Once the classification process is over, the next step is to perform the recognition process. The recognition process will done in two stages. First to identify the class in which the input image belongs to and second to identify the actual image from the class on which the method will give the maximum match.

The Overall recognition process is here represented by the help of an algorithm given as under. 


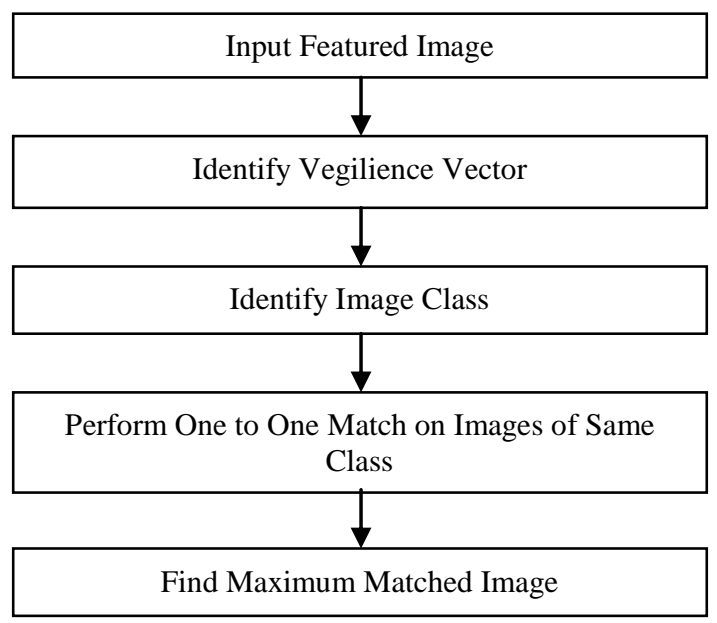

Fig. 6 : Recognition Process

The Overall recognition process is here represented by the help of an algorithm given as under.

Recognition(Classes, N,Img)

/* Classes is the ART classified N classes and Img is the input image been process for the work*

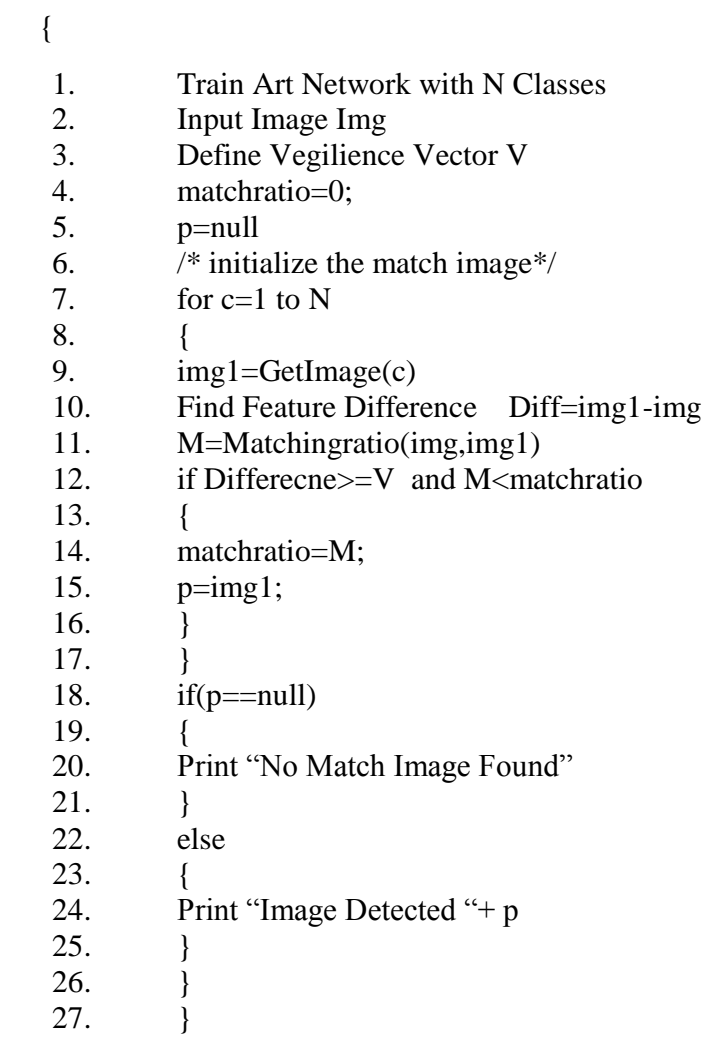

\subsection{Post Processing}

The post processing is performed to identify the matching ratio. The ratio here been analyzed based on the feature based analysis on each image of the specific class. A check is performed on all images in all classes. In result section, the matching ratio analysis is defined with obtained values.

\section{RESULTS}

The work is about to design a ART inspired classification model to perform the digital character recognition. The presented work is implemented under matlab 7.8. The work is tested for the normal image, noisy image and noise rectified image. The obtained results are presented in the form of graph.

\subsection{Setup 1}

Here in table 3, the parameters related to the work is defined. In this setup $\mathrm{N}$ number of images or equal size are trained using Art Network. As the peak vigilance value is taken, it generated the same number of classes of images.

Table 3 : Simulation Parameters

\begin{tabular}{|l|l|}
\hline Parameter & Value \\
\hline Number of Images & 26 \\
\hline Vigilance Value & 1 \\
\hline Images Type & Alphbet \\
\hline Image Size & $20 \times 40$ \\
\hline Image Fault/Noise & No \\
\hline Image Filteration Median & No \\
\hline Network Type & Art Network \\
\hline Input Image & A (Success Case) \\
\hline
\end{tabular}

Once the training set is generated the next work is to accept the user character image and perform the matching by using a simple image processing matching algorithm. It will match the image with each class and stores the matching ration. Here the results obtained from matching process are shown in table 4 . The results are presented in the increasing order or match ratio. It is observed that, the input character gives the maximum match value.

Table 4 : Matching Ratio

Q Matching Ratio $21.7391 \%$

M Matching Ratio $22.7778 \%$

Y Matching Ratio 23.3333\%

U Matching Ratio 25\%

V Matching Ratio 25\%

D Matching Ratio 26.2774\%

C Matching Ratio $28.2051 \%$

O Matching Ratio $28.3871 \%$

T Matching Ratio $29.8077 \%$

X Matching Ratio 31.3333\%

K Matching Ratio 31.9277\%

L Matching Ratio $32.6733 \%$

G Matching Ratio 33.9744\%

F Matching Ratio $34.2105 \%$

H Matching Ratio 34.4828\%

N Matching Ratio 35.8382\%

S Matching Ratio $36.4341 \%$

E Matching Ratio 39.1304\%

I Matching Ratio 40.7407\%

R Matching Ratio $40.8537 \%$

J Matching Ratio 41.3793\%

W Matching Ratio 43.662\% 
B Matching Ratio $44.6429 \%$

P Matching Ratio 48.062\%

Z Matching Ratio $57.9832 \%$

A Matching Ratio $99.1935 \%$

As shown in table 4 the most matching character is A. It shows that the input character and the matching character from database is same. It is showing the exact match for the character symbol. The results are also shown in the form of Bar Graph shown in Fig. 7.

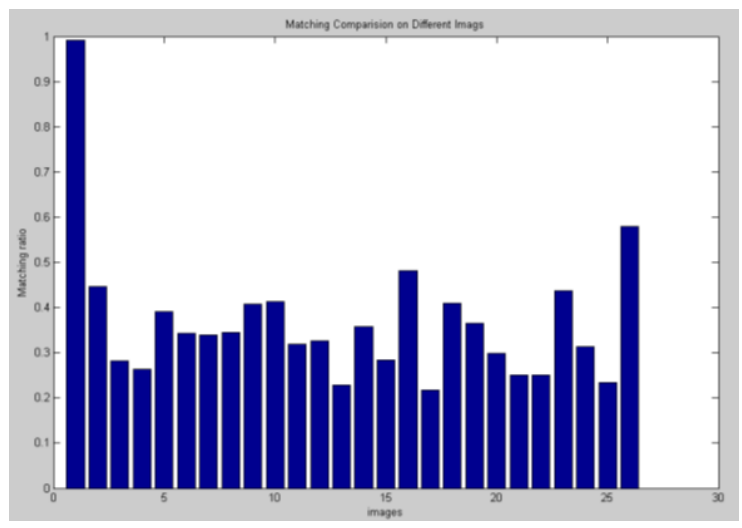

Fig. 7: Matching Ratio

As shown in Fig., the bar graph of the matching process. It shows the input Character "A" get the best match with same database character A.

\subsection{Setup 2}

The setup 2, is having the same parameters as of setup 1, the only difference is the considered case is the case of failure. In this setup input image is a diagonal line that does not perform match with any alphabetic character. The setup parameters are shown in table 5 .

\section{Table 5 : Simulation Parameter}

\begin{tabular}{|l|l|}
\hline Parameter & Value \\
\hline Number of Images & 26 \\
\hline Vigilance Value & 1 \\
\hline Images Type & Alphbet \\
\hline Image Size & $20 \times 40$ \\
\hline Image Fault/Noise & No \\
\hline Image Filteration Median & No \\
\hline Network Type & Art Network \\
\hline Input Image & Diagonal Line (Failure Case) \\
\hline
\end{tabular}

The work is performed on this wrong input image and the results obtained by the implementation process is shown in table 6 .

Table 6 : Setup 2 Matching Ratio

Matching Ratio

L Matching Ratio 0\%

C Matching Ratio $0.854701 \%$

K Matching Ratio $1.20482 \%$
X Matching Ratio 1.33333\%

V Matching Ratio 1.66667\%

Y Matching Ratio 1.66667\%

F Matching Ratio $2.63158 \%$

N Matching Ratio $2.89017 \%$

R Matching Ratio 3.04878\%

M Matching Ratio $3.33333 \%$

Q Matching Ratio 3.80435\%

H Matching Ratio $4.02299 \%$

B Matching Ratio $4.16667 \%$

E Matching Ratio $4.34783 \%$

U Matching Ratio $4.41176 \%$

G Matching Ratio $4.48718 \%$

T Matching Ratio $4.80769 \%$

Z Matching Ratio 5.04202\%

D Matching Ratio 5.10949\%

O Matching Ratio 5.16129\%

P Matching Ratio 5.42636\%

S Matching Ratio 5.42636\%

J Matching Ratio $5.74713 \%$

W Matching Ratio 6.33803\%

A Matching Ratio 8.06452\%

I Matching Ratio 8.64198\%

From the table, it is observed that the maximum match is performed for $\mathrm{I}$, as the input image is also a line placed diagonally. The maximum match value is only $8.6 \%$ that represents no image is matched to input image.

\subsection{Setup 3}

In this particular case, the noisy source image is considered as input. All the other parameters will remain same. The salt and pepper noise is been used to distorb the input image. The results obtained are shown in the form of bar graph shown in Fig. 8.

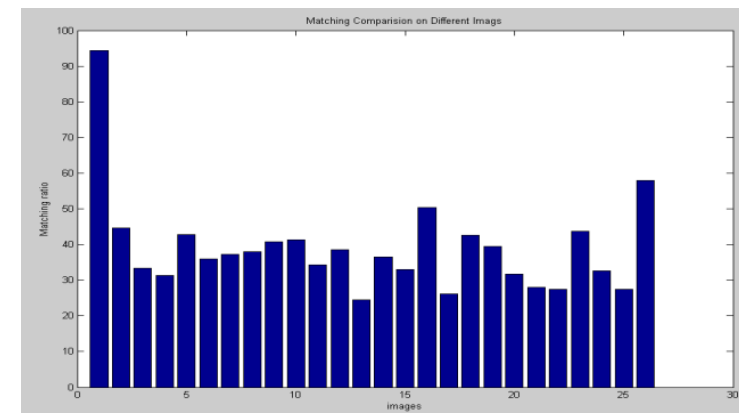

Fig. 8 : Matching Ratio Analysis

As shown in Fig., the bar graph of the matching process. It is observed that the input Character " $A$ " get the best match with Same database character $A$ with a matching ratio $91.3 \%$. It shows the work is effective even for the noisy image. 


\subsection{Setup 4}

In this setup, a noisy image is considered and used it for the identification process after imlementing the median filteration. All the other parameters are taken constant to the setup C. The obtained results are shown in Fig. 9.

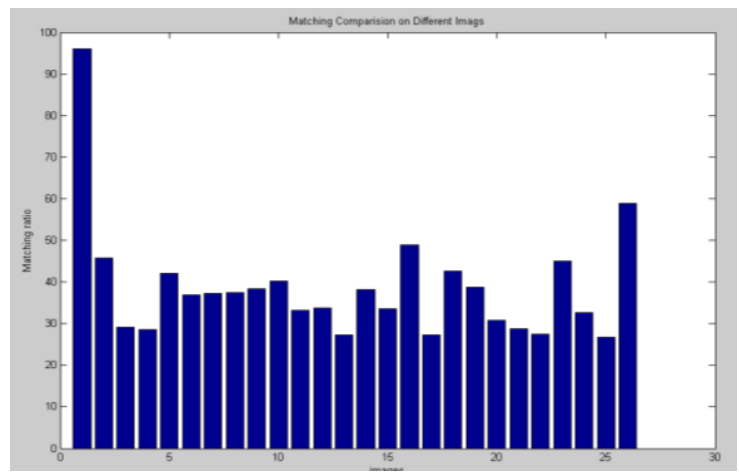

Fig. 9: Matching Ratio

As shown in Fig., the bar graph of the matching process. As the input character "A" get the best match with Same database character A with a higher ratio of $96 \%$. The results are obtained in case of noisy image that is been filtered by using median filter.

The presented work is effective enough to give successful results for normal image, noisy image and noisy filtered image. The image is giving higher matching ratio for successful case and low ratio for the failure case.

\section{CONCLUSION}

In this present work, the art network is implemented as the class specified based on feature mapping of image dataset. As the initial step the image database is being maintained to represent a different kind of character images. These images are trained using Art Network. The art network basically maintains the classes of dataset. Here, a higher accuracy level is driven to maintain the classes. As such threshold value given us almost same number of classes, as the number of images presented in the database. Once the database is trained the next work is about to perform the database matching based on input image. A level of distortion over the input image is taken to identify the matching ratio in case of error occurrence in images. In this work, the analysis is performed in the form of matching accuracy with and without fault in the input image and find the success and rejection ratio.

\section{REFERENCES}

[1] Jun Sun, "Low Resolution Character Recognition by Dual Eigenspace and Synthetic Degraded Patterns", HDP'04, November 12, 2004, Washington, DC, USA, pp 15-22

[2] Vinh Du Mai, "Building a License Plate Recognition System for Vietnam TollBooth", SoICT 2012, August 23 24, 2012, Ha Long, Vietnam, pp 107-114

[3] Jayakumar S Kandhasamy, "From Text to Images Through Meanings", ACE'04, June 3-5, 2004, Singapore, pp 222227

[4] Jonathan Holman, "Developing Usable CAPTCHAs for Blind Users", ASSETS'07, October 15-17, 2007, Tempe, Arizona, USA, pp 245-246

[5] Zhanghui Chen, "Effective Radical Segmentation of Offline Handwritten Chinese Characters towards Constructing Personal Handwritten Fonts", DocEng'12, September 4-7, 2012, Paris, France, pp 107-116

[6] Amritha Sampath, "Online Handwritten Character Recognition for Malayalam", CCSEIT-12, October 26-28, 2012, Coimbatore [Tamil nadu, India], pp 661-663

[7] Gurpreet Singh Lehal, "Choice of Recognizable Units for Urdu OCR”, DAR '12, December 16 2012, Mumbai, IN India, pp 79-85

[8] Dr.P.Subashini, "A combined preprocessing scheme for texture based Ice classification from SAR images", A2CWiC 2010, September 16-17, 2010, India, pp 1-6.

[9] Chergui Leila," ART NETWORK FOR ARABIC HANDWRITTREN RECOGNITION SYSTEM" 